\title{
Control of Modular Multilevel Converter based on Bridge Cells for 3-phase AC/AC Converter
}

\author{
Shin-ichi Hamasaki*, Kazuki Okamura* and Mineo Tsuji* \\ * Nagasaki University, 1-14 Bunkyo-machi, Nagasaki, 852-8521, Japan
}

\begin{abstract}
The Modular Multilevel Converter (MMC) with full bridge cells is available for a utility interactive inverter in high voltage line. When it is interconnected with power line, it is possible to control the active power flow in order to supply or charge the power in the line. This research applied a Hexagonal MMC to 3-phase AC-AC conversion system. The system is controlled by internal model principle and MMC can operate reliably. Theory of the power flow control of MMC and effectiveness of the proposed control method in the simulation are presented.
\end{abstract}

Index Terms -AC/AC power converter, Modular multilevel converter, Internal model principle

\section{INTRODUCTION}

Due to global warming, $\mathrm{CO}_{2}$ emission and abandoning nuclear generation, problems about energy and environment have been important. In this situation, renewable energy systems have been focused. Thus many kind of photovoltaic and wind power generation systems have studied in the world and it is expected to become increasingly popular in the future. However renewable energy generations give disturbance of voltage fluctuation to power grid because of their unstable power generation. Therefore the importance of interconnection equipment which control power flow in the grid will increase.

Meanwhile, the multilevel converter without transformer is developed. A modular multilevel converter (MMC)[1]-[9] is one of the transformer-less converter and applies to high voltage and high power conversion. The MMC is characterized by cascaded switching device modules with capacitors. The multilevel converter such as the MMC can improve waveform and reduce harmonic wave by multilevel output voltage. Thus it is able to operate with less harmonic disturbance and reduce filter volume. The triple star connected MMC[6] and the hexagonal type MMC[7][8] are constructed as a 3-phase AC/AC converter. The hexagonal type MMC can realize to reduce a number of cells. It can do power conservation regardless of voltage buck-boost and frequency. This topology is expected for a wide range of fields such as a wind power converter, a grid connection converter for power flow control and high voltage motor drive.

In this paper, the Hexagonal type MMC (H-MMC) is investigated and applied to grid connection converter and operate as a power flow controller. Control method of $\mathrm{H}$ MMC operated by AC/AC power conversion topology is described and the effectiveness of the proposed control is shown by simulation results in typical cases.

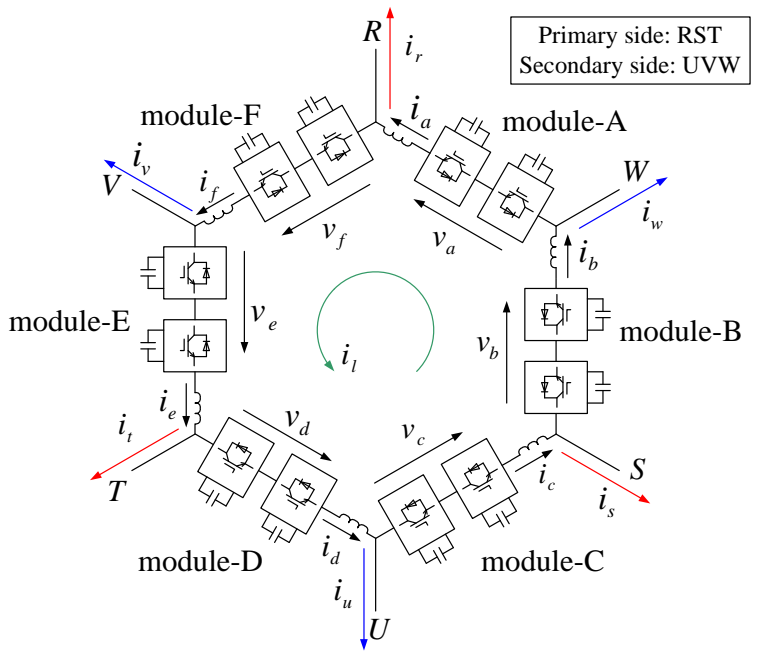

(a) The summary of H-MMC circuit

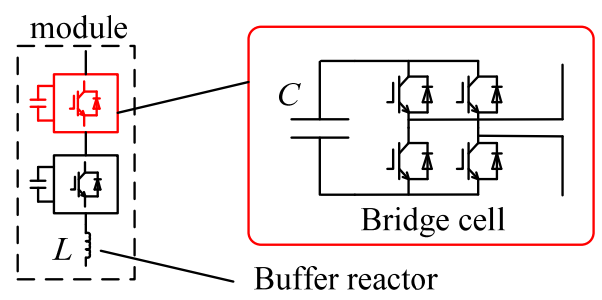

(b) Configuration of module and cell

Fig.1 circuit configuration of H-MMC

\section{CONFIGURATION OF CIRCUIT}

Fig.1 shows the configuration of H-MMC. The $\mathrm{H}$ MMC has six arm-modules connected in hexagonal shape. These modules consist of multiple cascade cells which have floating capacitors. Each arm module has a buffer reactor for preventing a short circuit and current regulation. The number of cells in an arm-module is 2 as shown in Fig.1(a). In this case, each full bridge cell can output positive and negative voltage. In Fig.1(a), terminals of R,S,T are primary side of power line, terminals of U,V,W are secondary side. Primary power via RST is converted to another frequency of voltage and secondary power is output via UVW. This configuration means that RSTdelta connection and UVW-delta connection overlap each other. Thus each module has primary and secondary lineto-line current. When the arm current is controlled, it is necessary that two frequency components are controlled at the same time. In this research, the internal model principle is applied to the current control. 


\section{CONTROL OF HeXAgOnAl TYPE MMC}

When H-MMC is applied to a power converter, it is able to convert frequency and voltage arbitrarily without transformer. At this time, currents of each arm-module must be controlled to be non-sinusoidal waveform, which is due to overlap line-to-line currents of primary and secondary. It is important that every capacitor voltage is managed to be constant and arm currents are exactly controlled without steady state error. In this study, capacitor voltages are kept by current control based on analysis the power flow in H-MMC.

\section{A. PQ-coordinate transformation}

The control of primary and secondary power is realized by the pq-coordinate transform. In particular, the primary power control is handled in the pq-current in order to adjust module capacitor voltage. Then the pqquantity is transformed to 3-phase quantity and the primary 3-phase quantity is controlled together with the secondary 3-phase current by the internal model principle. The pq-transform is defined as following equation.

$$
\left[\begin{array}{l}
F_{p} \\
F_{q}
\end{array}\right]=\sqrt{\frac{2}{3}}\left[\begin{array}{ccc}
\sin \omega_{0} t & \sin \left(\omega_{0} t-\frac{2 \pi}{3}\right) & \sin \left(\omega_{0} t-\frac{4 \pi}{3}\right) \\
\cos \omega_{0} t & \cos \left(\omega_{0} t-\frac{2 \pi}{3}\right) & \cos \left(\omega_{0} t-\frac{4 \pi}{3}\right)
\end{array}\right]\left[\begin{array}{l}
f_{u} \\
f_{v} \\
f_{w}
\end{array}\right](1)
$$

,where $F_{p}$ and $F_{q}$ are active and reactive component respectively. $f_{u}, f_{v}$ and $f_{w}$ are 3 -phase components. $\omega_{0}$ is an angular frequency the same as $u, v, w$-phase one.

\section{B. Power flow control in secondary side}

Power in secondary side can be controlled to arbitrarily value by using the pq-theory. The pq currents in secondary side are converted to 3-phase line-to-line currents, which are controlled on arm modules.

\section{Power flow control in primary side}

Power in primary side can be controlled in accordance with the pq-theory as well as the secondary power. In this control, however, the primary power is also controlled to maintain each capacitor voltage. The pq-current references in primary side are calculated from capacitor voltages in modules because there is relevance between the primary power and the capacitor voltage. Then power flows involved in arm-modules are analyzed. Voltages and currents in primary side are defined as (2)-(7).

$$
\begin{aligned}
& v_{r}=\sqrt{2} V_{1} \sin \omega_{1} t \\
& v_{s}=\sqrt{2} V_{1} \sin \left(\omega_{1} t-\frac{2}{3} \pi\right) \\
& v_{t}=\sqrt{2} V_{1} \sin \left(\omega_{1} t-\frac{4}{3} \pi\right) \\
& i_{r}=\sqrt{2} I_{1} \sin \left(\omega_{1} t-\phi_{1}\right) \\
& i_{s}=\sqrt{2} I_{1} \sin \left(\omega_{1} t-\frac{2}{3} \pi-\phi_{1}\right) \\
& i_{t}=\sqrt{2} I_{1} \sin \left(\omega_{1} t-\frac{4}{3} \pi-\phi_{1}\right)
\end{aligned}
$$

Secondary voltages and currents are defined as (8)-(13)

$$
\begin{aligned}
& v_{u}=\sqrt{2} V_{2} \sin \omega_{2} t \\
& v_{v}=\sqrt{2} V_{2} \sin \left(\omega_{2} t-\frac{2}{3} \pi\right) \\
& v_{w}=\sqrt{2} V_{2} \sin \left(\omega_{2} t-\frac{4}{3} \pi\right) \\
& i_{u}=\sqrt{2} I_{2} \sin \left(\omega_{2} t-\phi_{2}\right) \\
& i_{v}=\sqrt{2} I_{2} \sin \left(\omega_{2} t-\frac{2}{3} \pi-\phi_{2}\right) \\
& i_{w}=\sqrt{2} I_{2} \sin \left(\omega_{2} t-\frac{4}{3} \pi-\phi_{2}\right)
\end{aligned}
$$

,where $V_{1}$ and $I_{1}$ are RMS volumes in primary side, $V_{2}$ and $I_{2}$ are RMS volumes in secondary side. $\phi_{1}$ is the power factor angle in primary side, and $\phi_{2}$ is the power factor angle in secondary side.Each module has overlap components of primary and secondary as following equations.

$$
\begin{array}{lll}
v_{a}=v_{r}-v_{w}-l \frac{d i_{a}}{d t} & (14) & i_{a}=i_{r s}+i_{v w} \\
v_{b}=v_{w}-v_{s}-l \frac{d i_{b}}{d t} & (16) & i_{b}=i_{r s}+i_{w u} \\
v_{c}=v_{s}-v_{u}-l \frac{d i_{c}}{d t} & (18) & i_{c}=i_{s t}+i_{w u} \\
v_{d}=v_{u}-v_{t}-l \frac{d i_{d}}{d t} & (20) & i_{d}=i_{s t}+i_{u v} \\
v_{e}=v_{t}-v_{v}-l \frac{d i_{e}}{d t} & (22) & i_{e}=i_{t r}+i_{u v} \\
v_{f}=v_{v}-v_{r}-l \frac{d i_{f}}{d t} & (24) & i_{f}=i_{t r}+i_{v w}
\end{array}
$$

(14)-(25) show arm voltages and currents as shown in Fig.1(a). Each component is overlapped primary and secondary voltages and currents respectively. The average power of each arm-module is constructed by overlap of primary and secondary power, which is defined as $P_{A}$, $P_{B}, P_{C}, P_{D}, P_{E}, P_{F}$, respectively. These average powers are calculated by the following equations.

$$
\begin{aligned}
P_{A C E} & =P_{A}=P_{C}=P_{E} \\
& =\frac{V_{1} I_{1}}{\sqrt{3}} \cos \left(\phi_{1}-\frac{\pi}{6}\right)-\frac{V_{2} I_{2}}{\sqrt{3}} \cos \left(\phi_{2}-\frac{5 \pi}{6}\right) \\
P_{B D F} & =P_{B}=P_{D}=P_{F} \\
& =-\frac{V_{1} I_{1}}{\sqrt{3}} \cos \left(\phi_{1}-\frac{5 \pi}{6}\right)+\frac{V_{2} I_{2}}{\sqrt{3}} \cos \left(\phi_{2}-\frac{\pi}{6}\right)
\end{aligned}
$$

And the average power is calculated by (28).

$$
P_{X}=\frac{1}{T} \int_{t-T}^{t} p_{x} d t=\frac{1}{T} \int_{t-T}^{t} v_{x} i_{X} d t \quad(X=A-F, x=a-f)
$$

(26) and (27) show that modules A,C,E and modules B,D,F have the same average power $P_{A C E}$ and $P_{B D F}$ respectively. Following equations show sum of and difference between $P_{A C E}$ and $P_{B D F}$. 


$$
\begin{aligned}
& P_{A C E}+P_{B D F}=V_{1} I_{1} \cos \phi_{1}+V_{2} I_{2} \cos \phi_{2} \\
& P_{A C E}-P_{B D F}=\frac{1}{\sqrt{3}}\left(V_{1} I_{1} \sin \phi_{1}-V_{2} I_{2} \sin \phi_{2}\right)
\end{aligned}
$$

(29) shows the fluctuation of energy in H-MMC. If (29) is positive, all the capacitor voltages increase because the energy is stored in H-MMC. Otherwise, all the capacitor voltages decrease because the energy is emitted from $\mathrm{H}$ MMC. Therefore, it is desirable that (29) is maintained to 0 in order to operate H-MMC exactly at steady state. In this case, the following equation is obtained.

$$
V_{1} I_{1} \cos \phi_{1}=-V_{2} I_{2} \cos \phi_{2}
$$

(31) means that input active power in primary side is equal to the output active power in secondary side. When (31) is satisfied, the average voltage of all capacitors is maintained to constant.

On the other hand, (30) shows that the average power unbalance between modules A,C,E and B,D,F. This power unbalance affects to voltage unbalance of capacitor. Therefore it is desirable that (30) is maintained to 0 as well as the previous case. The following equation is obtained.

$$
V_{1} I_{1} \sin \phi_{1}=V_{2} I_{2} \sin \phi_{2}
$$

(32) means that the output reactive power between primary and secondary via H-MMC is the same value.

From the above, the average value of all capacitor voltage is controlled by the primary active power, and the average power difference is controlled by the primary reactive power respectively. The active and reactive currents have the relationship in (33)-(36). $I_{p 1}$ is primary side output active current, $I_{p 2}$ is secondary side output active current, $I_{q 1}$ is primary side output reactive current, and $I_{q 2}$ is secondary side output reactive current.

$$
\begin{aligned}
I_{p 1} & \propto I_{1} \cos \phi_{1} \\
I_{q 1} & \propto I_{1} \sin \phi_{1} \\
I_{p 2} & \propto I_{2} \cos \phi_{2} \\
I_{q 2} & \propto I_{2} \sin \phi_{2}
\end{aligned}
$$

The following control strategies are given by considering relevance between capacitor voltage and average power and applying (33)-(36) to (37) and (38).

$$
\begin{aligned}
& I_{p 1}^{*}=\left(K_{P 1}+\frac{K_{I 1}}{s}\right)\left(v_{C}-v_{C}^{*}\right)-\frac{V_{2}}{V_{1}} I_{p 2}^{*} \\
& I_{q 1}^{*}=\left(K_{P 2}+\frac{K_{I 2}}{s}\right)\left(v_{C-B D F}-v_{C-A C E}\right)+\frac{V_{2}}{V_{1}} I_{q 2}^{*}
\end{aligned}
$$

,where $v_{C}$ is average value of all capacitor voltages, $v_{C-A C E}$ is average capacitor voltage of modules $A, C, E, v_{C-B D F}$ is average capacitor voltage of modules $\mathrm{B}, \mathrm{D}, \mathrm{F}$, in addition, superscript * means reference value of each physical quantity.

In (37) and (38), first term of right-hand side means PI control of capacitor voltage and second term means feedforward term given by (33)-(36). The pq-currents are converted to three-phase currents and determine the lineto-line currents in primary side.

\section{Loop current control}

The primary side power control is able to balance the capacitor voltages between modules A, C, E and B, D, F. Voltage balance in all arm-modules is realized by a loop current control. Loop current has two frequencies of primary side and secondary side; if frequencies of primary side and secondary side are the same, loop current contains one frequency. The loop current reference is given by the following equation.

$$
\begin{aligned}
i_{l 1}^{*}= & \left(K_{P 3}+\frac{K_{I 3}}{s}\right)\left(v_{C}-v_{C-a b}\right) \sin \omega_{1} t \\
& +\left(K_{P 3}+\frac{K_{I 3}}{s}\right)\left(v_{C}-v_{C-c d}\right) \sin \left(\omega_{1} t-\frac{2}{3} \pi\right) \\
& +\left(K_{P 3}+\frac{K_{I 3}}{s}\right)\left(v_{C}-v_{C-e f}\right) \sin \left(\omega_{1} t-\frac{4}{3} \pi\right) \\
i_{l 2}^{*}= & \left(K_{P 3}+\frac{K_{I 3}}{s}\right)\left(v_{C}-v_{C-d e}\right) \sin \omega_{2} t \\
& +\left(K_{P 3}+\frac{K_{I 3}}{s}\right)\left(v_{C}-v_{C-f a}\right) \sin \left(\omega_{2} t-\frac{2}{3} \pi\right) \\
& +\left(K_{P 3}+\frac{K_{I 3}}{s}\right)\left(v_{C}-v_{C-b c}\right) \sin \left(\omega_{2} t-\frac{4}{3} \pi\right)
\end{aligned}
$$

,where $\omega_{1}$ is primary side angular frequency, $\omega_{2}$ is secondary side angular frequency. $\omega_{1} t$ is a base-phase which synchronize to $R S$-phase, and $\omega_{2} t$ is a base-phase which synchronize to $U V$-phase. And $v_{C-x y}(x, y=a-f)$ shows average voltage of each arm-module capacitor.

Fig. 2 shows a current vector plot at the case. This figure shows the primary side and the same principle can be applied to the secondary side. By the loop current control, the neutral point is intentionally displaced by (39) and (40). The displaced difference of the neutral point is the same as the loop current. In this way, the loop current control can adjust voltage balance between modules by unbalancing three-phase line-to-line current. Note that this unbalance affects only line-to-line current in $\mathrm{H}$ MMC. It means that phase currents of before and after the conversion are not affected by this unbalance. Hereafter the unbalanced line-to-line currents in H-MMC are shown with single quote.

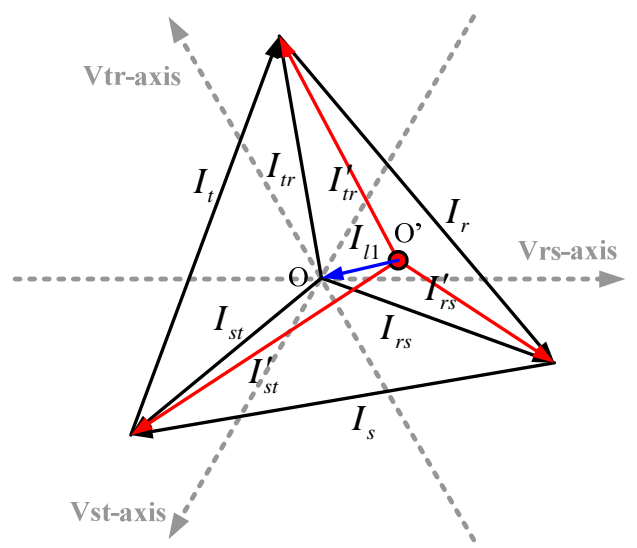

Fig.2 current vector plot (in primary side) 


\section{E. Internal model principle}

Power conversion and module voltage balance are controlled by regulating currents in H-MMC. Currents of modules are expressed by the following equations.

$$
\begin{aligned}
& i_{a}^{*}=i_{r s}^{*}+i_{v w}^{*}+i_{l}^{*}=i_{r s}^{*^{\prime}}+i_{v w}^{* \prime} \\
& i_{b}^{*}=i_{r s}^{*}+i_{w u}^{*}+i_{l}^{*}=i_{r s}^{*^{\prime}}+i_{w u}^{*^{\prime}} \\
& i_{c}^{*}=i_{s t}^{*}+i_{w u}^{*}+i_{l}^{*}=i_{s t}^{*^{\prime}}+i_{w u}^{*^{\prime}} \\
& i_{d}^{*}=i_{s t}^{*}+i_{u v}^{*}+i_{l}^{*}=i_{s t}^{*^{\prime}}+i_{u v}^{*^{\prime}} \\
& i_{e}^{*}=i_{t r}^{*}+i_{u v}^{*}+i_{l}^{*}=i_{t r}^{*^{\prime}}+i_{u v}^{*^{\prime}} \\
& i_{f}^{*}=i_{t r}^{*}+i_{v w}^{*}+i_{l}^{*}=i_{t r}^{*^{\prime}}+i_{v w}^{*^{\prime}}
\end{aligned}
$$

These show that each module current has both frequencies in primary and secondary side. Therefore it is difficult to control the current by the pq-transform. In this research, the module current is directly controlled by applying the internal model principle. The internal model principle has a merit that the applied controller can realize current control without steady error. It is defined that the open-loop transfer function of control system has mathematical model of reference signal. When $G_{C}(s)$ is the transfer function of controller which includes sinusoidal function model and $G_{G}(s)$ is considered as simplified model of the power system, the open-loop transfer function is expressed by (47)-(49).

$$
\begin{aligned}
& G_{C}(s)=\frac{K_{1} s}{s^{2}+\omega_{1}^{2}}+\frac{K_{2} s}{s^{2}+\omega_{2}^{2}} \\
& G_{G}(s)=\frac{1}{R_{G}+s L_{G}} \\
& G_{0}(s)=G_{C}(s) G_{G}(s) .
\end{aligned}
$$

,where $R_{G}$ is a resistance and $L_{G}$ is an inductance of the grid. The Laplace transform of cosine function is applied to controller because cosine function is better than sine function about phase margin.

The error of the reference $R(\mathrm{~s})$ and output $Y(\mathrm{~s})$ is written by (50) in a closed loop system.

$$
E(s)=R(s)-Y(s)=\frac{R(s)}{1+G_{0}(s)}
$$

Here $R(\mathrm{~s})$ is defined as the following sinusoidal function,

$$
R(s)=\frac{A_{1} \omega_{1}}{s^{2}+\omega_{1}^{2}}+\frac{A_{2} \omega_{2}}{s^{2}+\omega_{2}^{2}}
$$

According to the theorem of final value, (52) is obtained.

$$
\lim _{t \rightarrow \infty} e(t)=\lim _{s \rightarrow 0} s E(s)=0
$$

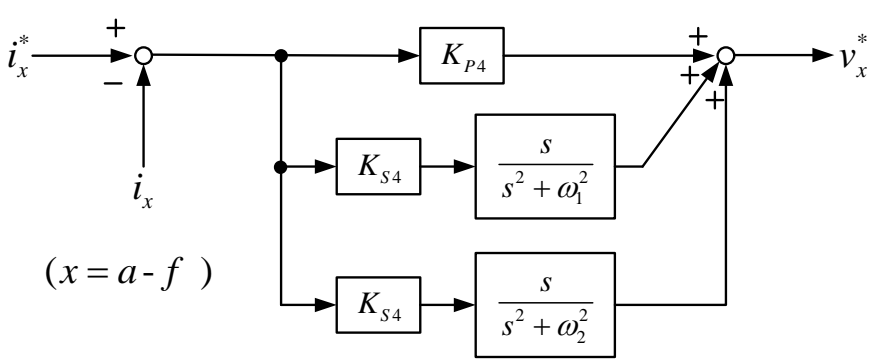

Fig.3 block diagram of current control

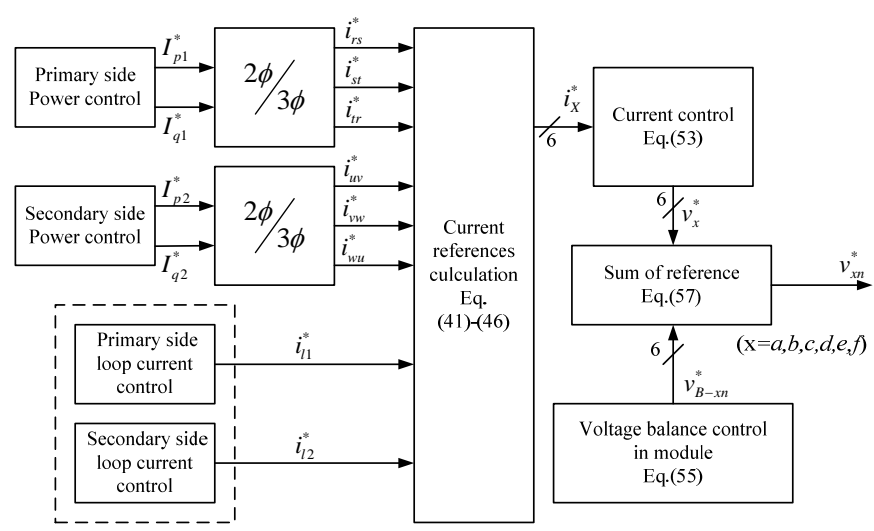

Fig.4 configuration of total control system

(52) shows that the error at steady state becomes 0 by using the internal model principle.

Fig.3 shows a block diagram of controller applied the principle and (53) shows the control strategy.

$$
v_{x}^{*}=K_{P 4}\left(i_{x}^{*}-i_{x}\right)+K_{S 4}\left(i_{x}^{*}-i_{x}\right)\left(\frac{s}{s^{2}+\omega_{1}^{2}}+\frac{s}{s^{2}+\omega_{2}^{2}}\right)
$$

First term of the right side is a proportional control, and second term is a control of the internal model principle. The controller has sinusoidal internal model of primary and secondary frequencies.

\section{F. Voltage balance in each module}

Voltage balance in each module is introduced[1]. Because unbalance in each arm module is occurred due to variation of individual differences of capacitance, switching delay and so on. The balancing control is given by (54).

$$
\begin{aligned}
& v_{B-x n}^{*}=-K_{P 5}\left(\bar{v}_{C x}-v_{C-x n}\right) i_{x} \\
& \bar{v}_{C x}=\frac{\sum_{n=1}^{N} v_{C-x n}}{N}
\end{aligned}
$$

(55) is an average value of module capacitor voltage, $N$ is the number of cells. In this case, $N=2$ is applicable from Fig.1. $v_{C-x n}$ is capacitor voltage, $v_{B-x n}^{*}$ is voltage reference value for balancing capacitor voltage in each module. Index '- $x n$ ' means $n$-th cell on $x$-module. This control can adjust voltage balance among cells in each module because of exchange of power among cells. The sum of this control reference value is shown by (56). 

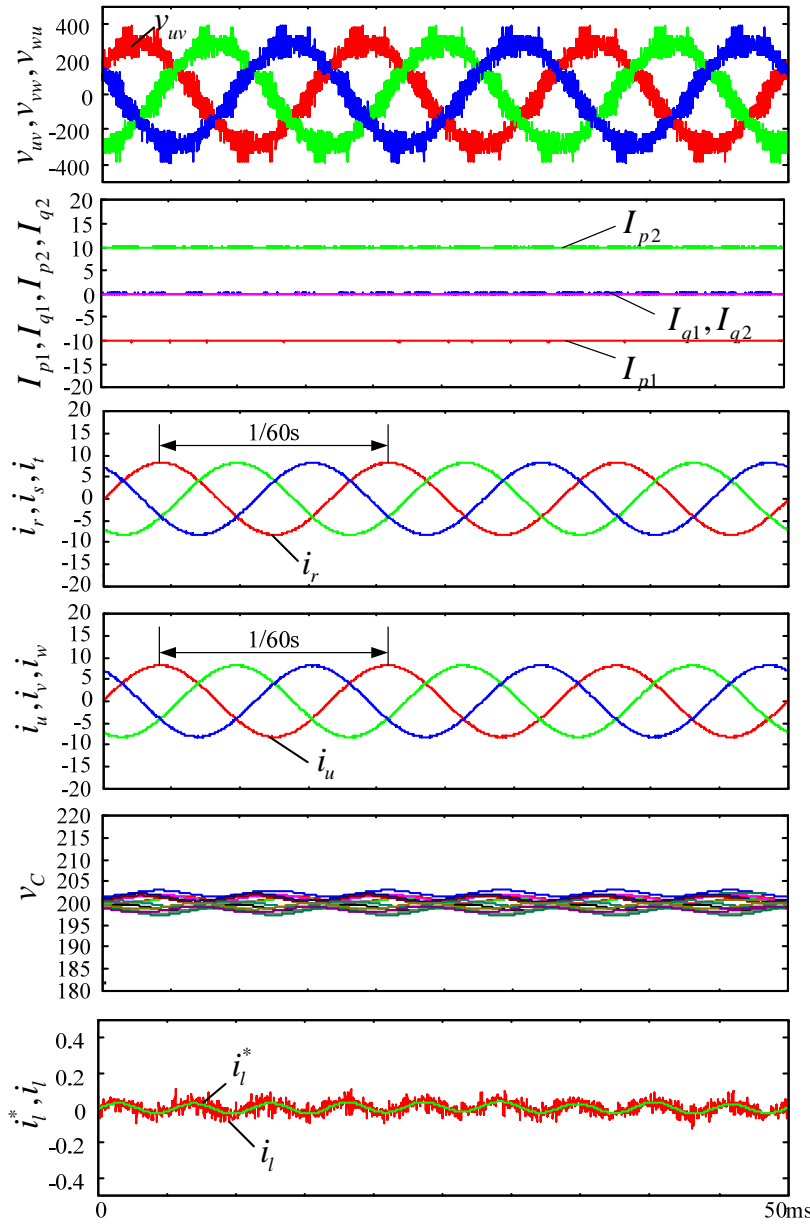

Fig.5 simulation result of the same frequency $(60 \mathrm{~Hz})$

$$
\sum_{n=1}^{N} v_{B-x n}^{*}=0
$$

(56) implies that this control does not affect the current control. Voltage reference values summed up from (53) and (54) are outputted from each cell.

$$
v_{x n}^{*}=v_{x}^{*}+v_{B-x n}^{*}
$$

The configuration of overall control system is shown in Fig.4.

\section{Simulation}

Simulation is performed to verify the proposed control methods by using the circuit in Fig.1. Table 1 shows the parameters of the circuit, and table 2 shows the control gains. In this research, the H-MMC has 2 full-bridge cells per an arm, and each cell is operated the uni-pole PWM switching. The H-MMC can realize multilevel voltage output by the PWM switching pattern. In the PWM switching, a carrier wave is triangular wave of which phase is shifted according to the number of modules. The line voltage becomes 9-levels in case of 4 cells in line-toline modules.
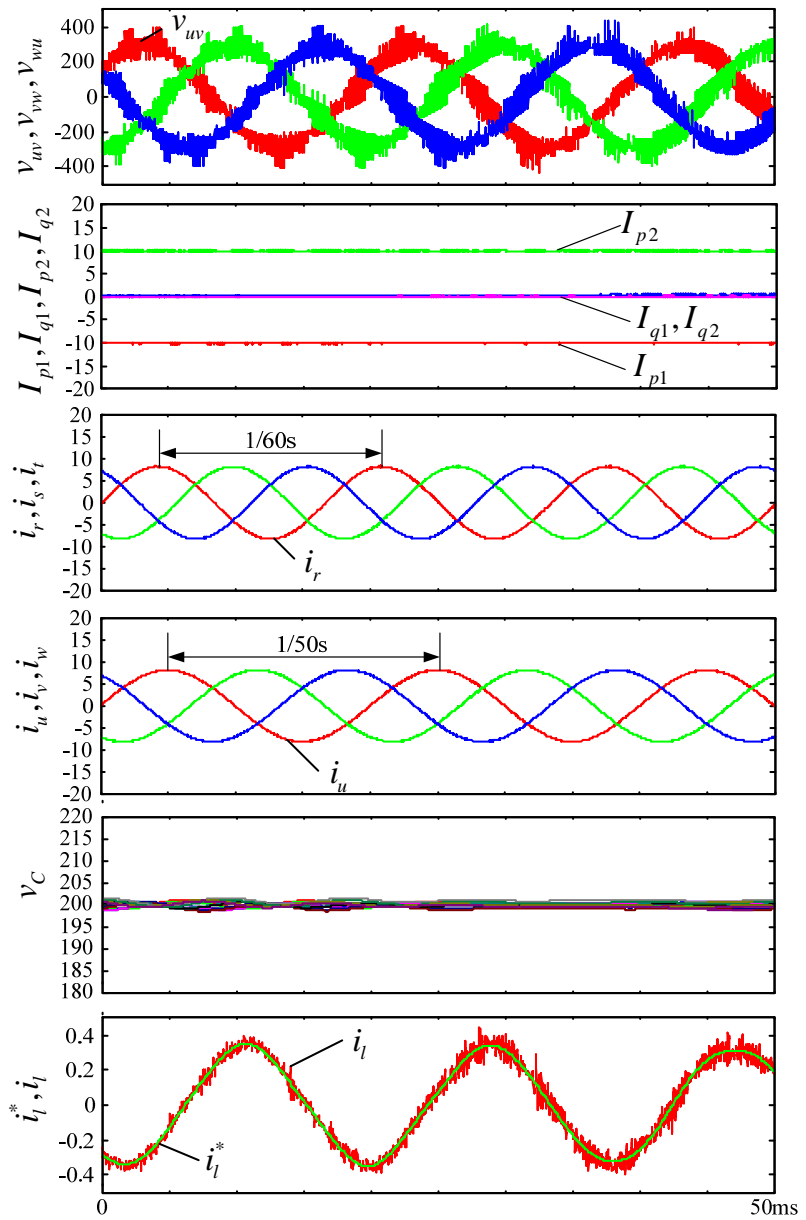

Fig.6 simulation result of different frequencies $(60-50 \mathrm{~Hz})$

TABLE 1. Parameters of circuit

\begin{tabular}{|c|c|c|c|}
\hline$V_{1}$ & $200 \mathrm{~V}$ & $V_{2}$ & $200 \mathrm{~V}$ \\
\hline$l$ & $5 \mathrm{mF}$ & $C$ & $5000 \mu \mathrm{F}$ \\
\hline$v_{C}{ }^{*}$ & $200 \mathrm{~V}$ & $L$ & $3 \mathrm{mH}$ \\
\hline
\end{tabular}

TABLE 2. Gains of control

\begin{tabular}{|c|c|c|c|}
\hline$K_{P 1}$ & 1.5 & $K_{I 1}$ & 5.0 \\
\hline$K_{P 2}$ & 1.5 & $K_{\mathrm{I} 2}$ & 5.0 \\
\hline$K_{P 3}$ & 0.5 & $K_{I 3}$ & 1.0 \\
\hline$K_{P 4}$ & 40.0 & $K_{S 4}$ & 13000.0 \\
\hline$K_{P 5}$ & 0.1 & \multicolumn{2}{|l}{} \\
\hline
\end{tabular}

Fig.5 shows a simulation result that input and output frequencies are $60 \mathrm{~Hz}$. It is confirmed that output line-toline voltages of secondary side are multilevel. Focusing attention to the pq-quantity, $I_{p 1}$ and $I_{p 2}$ are the same value $10 \mathrm{~A}$ but opposite sign. This means that received active power of H-MMC from primary side is the same value as the supplied active power to secondary side. As a result, average voltage of all capacitors is maintained to constant value. On the other hand, $I_{q 1}$ and $I_{q 2}$ have same value. Thus, voltage between module A,C,E and B,D,F is also adjusted. Because currents in primary and secondary side are the same values in case of the same frequency. 


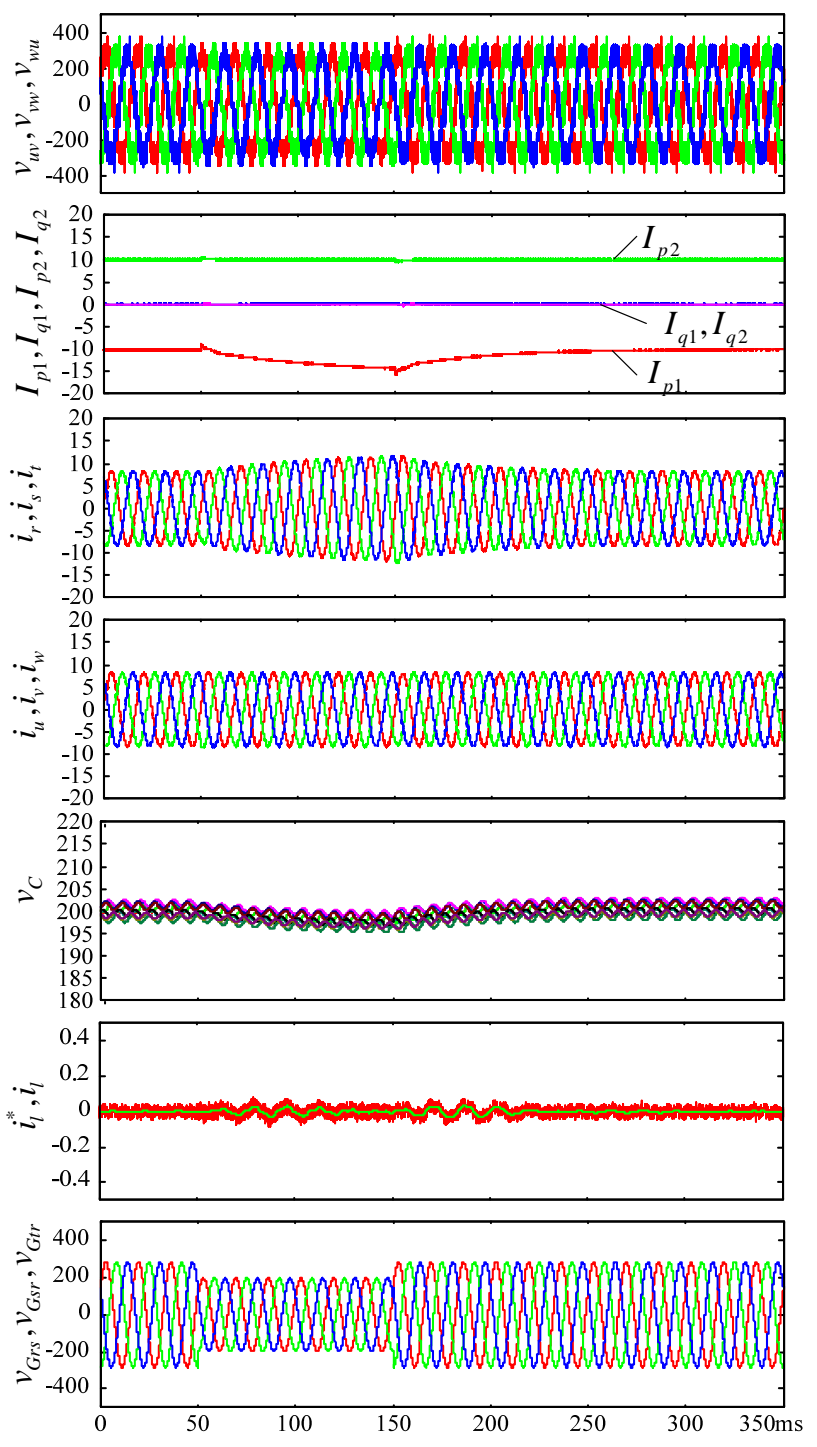

Fig.9 simulation result of momentary voltage drop

Fig.6 shows a simulation result that input and output frequencies are different. Primary side frequency is $60 \mathrm{~Hz}$ and secondary frequency is $50 \mathrm{~Hz} . I_{p 1}$ and $I_{p 2}$ have the same value 10A with opposite sign. Even if frequency is converted, if the same value of voltage and current, $I_{p 1}$ and $I_{p 2}$ have the same value regardless of frequency. And the secondary side current is converted to $50 \mathrm{~Hz}$ exactly. The loop current has large amplitude because of overlap of two frequencies.

Fig. 9 shows a simulation result that momentary $25 \%$ voltage drop occurs during $50-150 \mathrm{~ms}$. While the voltage drop occurs, capacitor voltages decrease and $I_{p 1}$ decrease, either. When $I_{p 1}$ is adjusted negative value, the capacitor voltages increase because of more active power inflow. Therefore amplitude of primary currents becomes large. However currents in secondary side are not affected by this power fluctuation. The proposed scheme is strong to the voltage fluctuation because control strategy is independent in the primary side and the secondary side.

These simulation results show that H-MMC is able to convert exactly regardless of the frequency and voltage. The H-MMC can be applied to grid connection converter as a power flow controller and a power conditioner for wind generators.

\section{CONCLUSION}

This paper proposed a new control of MMC for 3phase AC/AC converter .The H-MMC is applied to grid connection system as the power flow controller, and proposed how to control the voltage and the power flow of the H-MMC. In the H-MMC, sinusoidal currents are controlled directly by using the internal model principle. The simulation demonstrated that the proposed control method is able to control exactly and have a good performance.

\section{ACKNOWLEDGEMENTS}

This work is supported by JSPS KAKENHI (Grant-inAid for Young Scientists (B)) Grant Number 24760237.

\section{REFERENCES}

[1] M. Hagiwara, H. Akagi, “Control and Experiment of PulsewidthModulated Modular Multilevel Converters”, IEEE Trans. on PE, Vol.24, No.7, pp.1737-1746, 2009.

[2] M. Hagiwara, R. Maeda, and H. Akagi, “Theoretical Analysis and Control of the Modular Multilevel Cascade Converter Based on Double-Star Chopper-Cells (MMCC-DSCC)"(in Japanese), IEEJ Trans. on IA, Vol.131, No,.1, pp.84-92, 2011.

[3] M. Glinka, R. Marquardt, "A New AC/AC Multilevel Converter Family”, IEEE Trans. on IE, Vol.52, No.3, pp. 662-669, 2005.

[4] R.Marquardt,"Modular Multilevel Converter:An universal concept for HVDC-Networks and extended DC-Bus-applications”,The Internationa Power Electronics Conference(IPEC),pp.502-507,2010.

[5] H. Fujita, M. Hagiwara, H. Akagi, "Power Flow Analysis and DCCapacitor Voltage Regulation for the MMC-DSCC”(in Japanese), IEEJ Trans. on IA, Vol.132, No.6, pp.659-665, 2012.

[6] W. Kawamura, H. Akagi, "Control Strategy of a Modular Multilevel Cascade Converter Based on Triple-Star Bridge-Cells (MMCC-TSBC)”, The Institute of Electrical Engineers of Japan, 1-49, pp.241-246, 2012

[7] L. Baruschka, A. Mertens, "A new 3-phase AC/AC modular multilevel converter with six branches in hexagonal configuration”, Energy Conversion Congress and Exposition (ECCE), IEEE, pp.4005-4012, 2011

[8] T. Hosaka, K Akiba and H Fujita, ”A Unified Power-flow controller Consisting of Six Cascaded H-Bridge Converters”, The Institute of Electrical Engineers of Japan, 1-36, pp.247-250, 2011

[9] S. Fukuda, T. Yoda "A Current Control Method for Active Filters Using Sinusoidal Internal Model”, T.IEEJ, Vol.120-D, No.12, pp.140-146, 2000

[10] S. Hamasaki, M. Tsuji, E. Yamada "A Study on Power Flow Control for Distributed Generator with EDLC”, SYMPOSIUM ON POWER ELECTRONICS, ELECTRICAL DRIVES, AUTOMATION AND MOTION (SPEEDAM), Vol.1, pp.1502-1507, 2010.

[11] S. Hamasaki, K. Okamura, M. Tsuji, "Power Flow Control of Modular Multilevel Converter based on Double-Star Bridge Cells Applying to Grid Connection” ICEMS2012,No.DS1G4-9, pp.1-6, 2012.10 